\title{
Deceptive Slanders of Cardiovascular Pathology in Covid-19 Ethnic Minorities
}

\author{
Sayed Nour* \\ Department of Pediatric Cardio Surgeon, Orleans, France
}

*Corresponding author: Sayed Nour, Consultant Pediatric Cardio Surgeon, Independent Cardiovascular Researcher, Le

LAB'O, Orleans Technopole, 1 avenue du Champs de Mars, 45074, Orleans, France

\begin{tabular}{|c|c|}
\hline ARTICLE INFO & ABSTRACT \\
\hline 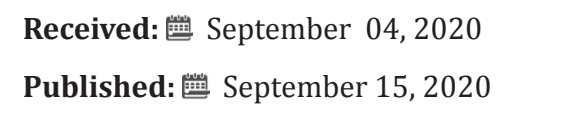 & $\begin{array}{l}\text { Reviewing the Covid-19 literature over the past six months we can identify pervasive } \\
\text { endothelial dysfunction disorders, whether in the form of comorbid conditions e.g. } \\
\text { arterial hypertension, mediated by pathogens, e.g., thromboembolic syndrome and / or }\end{array}$ \\
\hline $\begin{array}{l}\text { Citation: Sayed Nour. Deceptive Slanders } \\
\text { of Cardiovascular Pathology in Covid-19 } \\
\text { Ethnic Minorities. Biomed J Sci \& Tech Res } \\
\text { 30(3)-2020. BJSTR. MS.ID.004945. }\end{array}$ & $\begin{array}{l}\text { unless you are young and slim and Caucasian, we cannot cure you!Indeed, it is totally } \\
\text { unacceptable in the twenty-first century to involve racial or ethnic assumptions in science } \\
\text { without providing substantial evidence, especially in renowned journals. The question } \\
\text { is, do we realize the extent of the psychological damage we are causing to individuals } \\
\text { belonging to these ethnic minorities, to their wives, children and friends ...?We aim } \\
\text { through the present work to correct this erroneous thought, as well as to expose our } \\
\text { visions concerning the management of Covid-19, which unfortunately became a politico- } \\
\text { mediatic subject and remains without effective solution. }\end{array}$ \\
\hline
\end{tabular}

\section{Introduction}

In a post-mortem study of 10 African American patients with COVID-19, Fox and colleagues claimed key pathological states in this demographic, e.g. right ventricular (RV) dilatation [1]. Meanwhile, they have mentioned normal cardiac dimensions $(3.4 \mathrm{~cm}$ and $3.6 \mathrm{~cm}$ ) and presented a normal heart, showing a well trabeculated right ventricular free wall, which is the most vulnerable part that cannot withstand intracardiac pressure. Thereafter, in less than a month the authors published a new series, in which they had faded their exclusive identification of cardiac risk factors in African American population and maintained claiming RV dilation in Covid 19 [2]. It is important to precise that all these patients presented endothelial dysfunction comorbidities, linked to stressful socioeconomic status [3].

All studies on ethnic minorities lack comparative data with Caucasian groups, subjected to similar environmental conditions. Genetics rather than ethnicity, is most rational Vector in Science, for example, difficulties of ventilators weaning in Noonan or Down syndrome. We stitch wounds for better healing, which is an endothelial function process. We know how to avoid sunrays in dark skin or absorbable stitches in Down syndrome to avoid undesired results. But we do not add corrosives and complain about keloids (fibrosis). Only endothelial function tests in multi-ethnic infants, can refute or confirm these claims. The ugly fact is that we are still blowing up the most important circulatory driving force that controls hemodynamic and microcirculation: the heaven's door of cellular biology and tissue oxygenation of vital organs, heart included.

Surgically, we can bypass the right or left ventricle but with the permission of the almighty respiratory pump [4]. As a reminder, endothelium is the precursor of the cardiovascular system, depends on shear stress of bloodstream to maintain its functions like vascular tone, coagulation, angiogenesis, apoptosis, diabetes, atherosclerosis, immune system, inflammatory response, nitric monoxide synthesis, etc. Endothelial shear stress (ESS) controls vasculogenesis, cardio genesis, embryogenesis, organogenesis through the angiogenesis-apoptosis interdependency process, from the 8th day of gestation until death. An imbalanced angiogenesisapoptosis interdependency can induce irreversible cellular 
damages like Eisenmenger syndrome, Cor pulmonale or heart failure: cardiomyocytes apoptosis, compensated by angiogenic hypertrophy or fibrotic dilatation [5].

In antenatal, the right ventricle (RV) is the main trigger of ESS and moderator of foetal development even in severe cardiomyopathies, e.g., hypoplastic left heart syndrome. It distributes blood flow to the left ventricle (LV) through the foramen ovale, to pulmonary and collapsed lungs circuit $(\approx 10 \%)$ and to the descending aorta through the ductus arteriosus. In the postnatal period, with shunts closures, the respiratory pump becomes a key circulatory driving force to deal with the massive blood volume at the rightheart side $(\geq 80 \%)$. It becomes the main trigger of ESS to continue cardiovascular remodelling e.g., increasing LV mass in maintaining low remodelling at the right-heart side ( $\mathrm{RV} / \mathrm{LV}$ mass $\approx 1 / 6)$. It squeezes the pulmonary parenchyma in an accordion-like manner, releasing plenty of endothelial mediators to drop the pulmonary vascular resistances (PVR), to improve hemodynamic as well as tissue oxygenation with first breath after birth. By controlling the pulmonary afterload, the respiratory pump controls RV preload and cardiac output (Frank- Starling law), helped with other influential forces like the muscle pumps, gravity, atmospheric pressure... [6]. Disturbed RV preload provokes cardiovascular disorders like in astronauts, professional scuba divers, sleep apnea... and serious complications in bedridden ventilated patients. The respiratory pump which is a low-pressure momentarily closed hydraulic circuit, due to the epiglottis effect, must deal with two types of fluids: the compressible Newtonian (air) and the incompressible nonNewtonian (blood); and a delicate alveolar system composed of two types of single-cell layers: the epithelium and the endothelium, to ensure gas exchanges. In addition, the extra-alveolar and alveolar endothelial cells have different embryological origins and behaviour, e.g., drawbacks of inhalational PAH therapies [7].

Furthermore, we have proved that the pulmonary endothelium, which is unexploited before birth, responds differently and more effectively to ESS stimuli, compared to left-heart side endothelium [8]. Then we come with the ventilator's invasion and neuromuscular blockades to transform the respiratory pump into a piston-like, closed, pressurized, caged, purulent, ...hydraulic circuit. Maintenance of endogenous pulmonary endothelial mediators is mandatory to respiratory or more correctly metabolically, distressed patients, whatever the underlaying pathology. Apparently, once the pulmonary production of ESS is compromised due to pathological conditions of the contractile structures of the respiratory pump e.g., pneumonia, oedema, patients exhibit symptoms and signs like: dyspnoea, tachypnoea, tachyarrhythmia, orthopnoea... even with very mild hypoxia (Sp02 $\leq 94 \%)$, which is normally uncompromising for life. This means desperate requirements of endogenous pulmonary mediators for hemodynamic and metabolic improvements.
It is all about how to fully engage the respiratory pump and its influential forces, the gravitational effect. For example, pulmonary ESS enhancement is the hallmark of physical exercise, exhibited without shortness of breath in marathon runners due to the "second wind" effect. In contrast, an overweight nonathlete runner exhibits shortness of breath and leans forward with hands on knees and not in a recumbent position. On the other hand, a congestive heart failure patient, in a recumbent position exhibits a nocturnal orthopneic dyspnoea to improve hemodynamic. Or patients in severe cyanosis (SpO2 $\leq 80 \%$, exhibits squatting position without shortness of breath. This is exactly what lies behind the untold explanations of studies showing the advantages of non-invasive ventilation, low-dose neuro blockade: maintaining chest wall recoil; prone position, obesities: correlated with diaphragmatic compression by stagnant hepato splanchnic venous capacity; tracheostomy: decreasing interalveolar pressure by reducing airflow energy losses and tracheal dead space, etc.

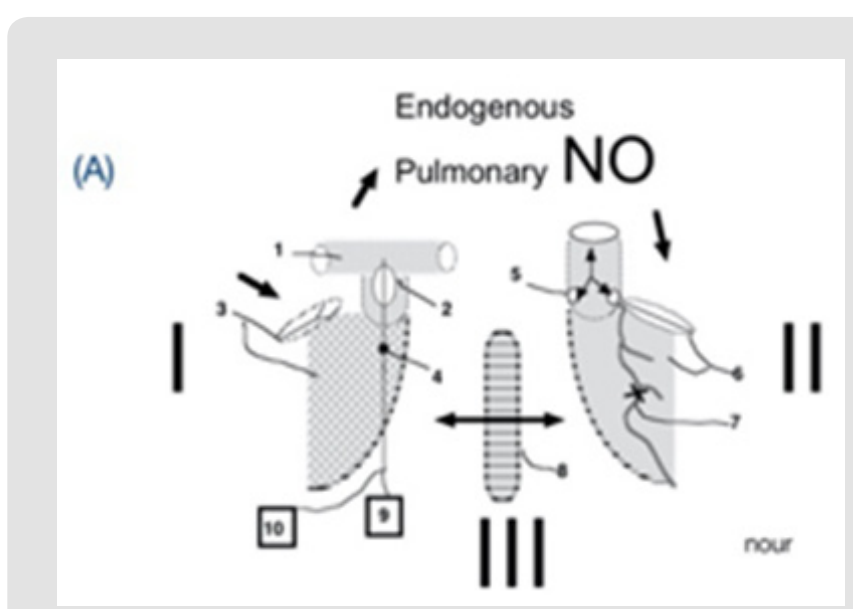

Figure 1A: Presumed mechanism and passage of induced pulmonary eNOS. 1 = Pulmonary artery (PA); 2 = pulsatile catheter fitting PA trunk; 3 = right ventricle (RV) inletoutlet compartments; $4=$ infundibular site of pulmonary catheter insertion; $5=$ arrows showing presumed passage of pulmonary eNOS (backward through coronary ostia and/or forward through systemic circulation); $6=$ left ventricle (LV) inlet-outlet compartments; $7=$ permanent ligation of the left anterior descending coronary artery distal to the second diagonal branch; $8=$ interventricular septum; 9 = cardiorespiratory monitor; $10=$ pneumatic driving force. $\mathrm{I}=$ pulmonary eNOS primarily induced at PA zone with catheter pulsation; II = pulmonary eNOS natural passage through the left heart circuit; III = presumed pulmonary eNOS involvement in myocardial recovery most probably through microcirculation and/or the RV interseptal coronary network.

Besides, vasopressors and the encounter of circulatory assist devices (CAD) with the circulatory system creates vicious circle of endothelial dysfunction and momentum energy losses. As it is known, boundary wall friction of bloodstream inside rigid narrow 
conduits of CAD provokes the post cardiotomy syndrome [9], posthaemodialysis pains [10]. Similarly, pressurized airflow inside ventilators rigid conduits but with different diameters promotes barotrauma and reinfection by Venturi effect. We think it is time to have a deep breath and calm down, through the respiratory pump, and let it do the knocking on heaven's door instead of our unfortunate patients. We did not need a well performing heart pump (Figures 1A-1D) [11], or even heartbeats, ventilators, and pharmacological supports $[12,13]$, to improve hemodynamic in fragile animal models, but endogenous pulmonary endothelial mediators. This is not polemical or science fiction work, it is what we have developed over the past two decades with pioneering doctors and legendary heart surgeons, including the former president of a well-known Academy of Sciences.
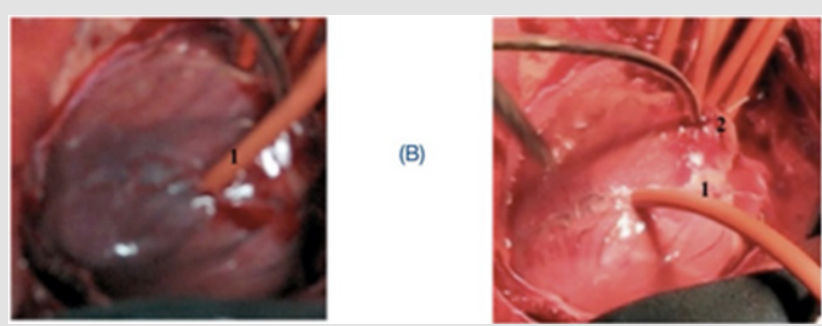

Figure 1B: Macroscopic reduction of the ischemic zone. Left panel figure showing dark infarcted myocardial after $50 \mathrm{~min}$ of ischemia; Right panel figure showing significant reduction of ischemic myocardial zone after $10 \mathrm{~min}$ of pulsation; 1 = left anterior descending coronary artery snugger; 2 = infundibular site of the intrapulmonary pulsatile catheter insertion.
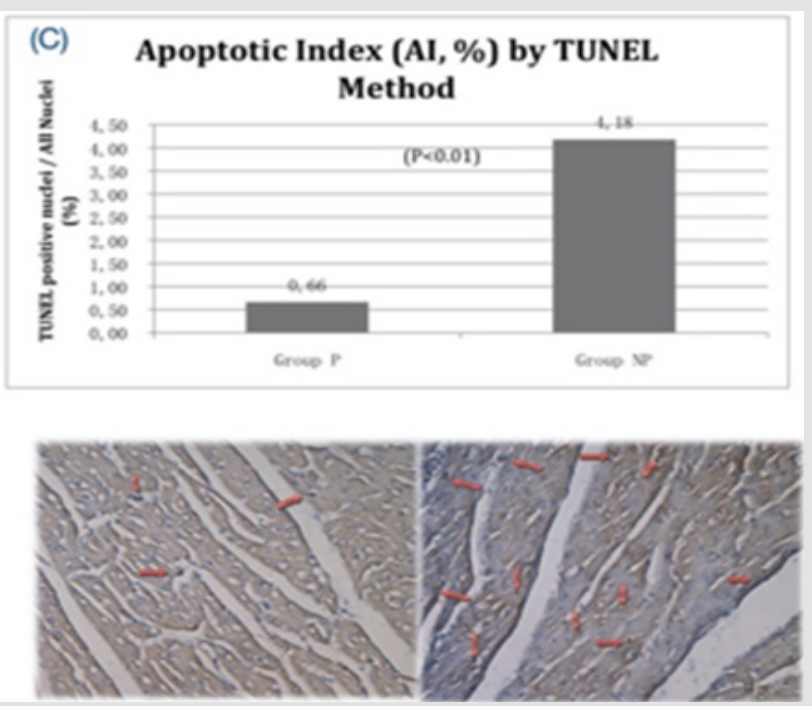

Figure 1C: Myocardial apoptosis by TUNEL technique. Upper panel, apoptotic index (AI) in both groups. AI in group $\mathrm{P}$ was significantly lower than that in group $\mathrm{NP}(\mathrm{P}<0.01)$; lower panel, representative figures from both groups showing apoptotic cells manifestations (red arrows): lower left, group P, lower right, group NP.

\section{(D) Myocardial eNOS Expression (RT-PCR)}

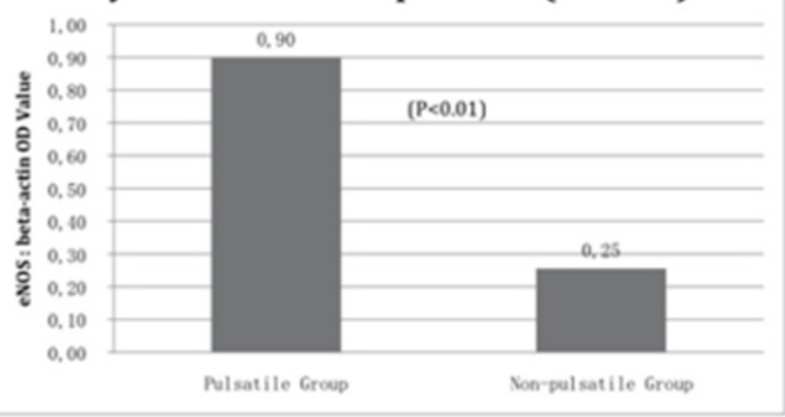

Figure 1D: Myocardial eNOS mRNA expression. RT-PCR results shown with statistics, in which myocardial eNOS expression was significantly higher in group P compared to group NP $(\mathrm{P}<0.01)$. Pulsatile group $(\mathrm{P})$, Non-pulsatile group (NP).

\section{References}

1. Fox SE, Akmatbekov A, Harbert JL, Li G, Quincy Brown J, et al. (2020) Pulmonary and cardiac pathology in African American patients with COVID-19: an autopsy series from New Orleans. Lancet Respir Med 8(7): 681-686.

2. Fox SE, Li G, Akmatbekov A, Harbert JL, Lameira FS, et al. (2020) Unexpected Features of Cardiac Pathology in COVID-19 Infection. Circulation.

3. Sundquist J, Malmström M, Johansson SE (1999) Cardiovascular Risk Factors and the Neighbourhood Environment: A Multilevel Analysis. Int J Epidemiol 28(5): 841-845.

4. Beke DM (2016) Norwood Procedure for Palliation of Hypoplastic Left Heart Syndrome: Right Ventricle to Pulmonary Artery Conduit vs Modified Blalock-Taussig Shunt. Crit Care Nurse 36(6): 42-51.

5. Petrovic D, Zorc-Pleskovic R, Zorc M (2000) Apoptosis and proliferation of cardiomyocytes in heart failure of different etiologies. Cardiovasc Pathol 9(3): 149-152.

6. Nour S, Wu G, Zhensheng Zh, Juan CC, Alain C, et al. (2009) The forgotten driving forces in right heart failure: concept and device. Asian Cardiovasc Thorac Ann 17(5): 525-530.

7. Stevens T, Sem P, Maria GF, Diego A, Erica H, et al. (2008) Lung vascular cell heterogeneity: endothelium, smooth muscle, and fibroblasts. Proc Am Thorac Soc 5(7): 783-791.

8. Nour S, Dai G, Carbognani D, Feng M, Lila N, et al. (2012) Intrapulmonary shear stress enhancement: A new therapeutic approach in pulmonary arterial hypertension. Pediat Cardiol 33(8): 1332-1342.

9. Brettner F, Chappell D, Schwartz L, Alexander L, Philipp K, et al. (2017) Vascular Endothelial Dysfunction During Cardiac Surgery: On-Pump Versus Off-Pump Coronary Surgery. Eur Surg Res 58(5-6): 354-368.

10. Davison SN (2003) Pain in Hemodialysis Patients: Prevalence, Cause, Severity, and Management. Am J Kidney Dis 42(6):1239-1247.

11. Nour S, Dai G, Wang Q Fei W, Juan CC, et al. (2012) Forgotten driving forces in right heart failure (Part II): Experimental study. Asian Cardiovasc Thorac Ann 20(6): 646-657.

12. Nour S, Carbognani D, Chachques JC (2017) Circulatory flow restoration versus cardiopulmonary resuscitation: new therapeutic approach in sudden cardiac arrest. Artif Organs 41(12): 356-366.

13. Nour S, Daya Y, Gong D, Lila N, Juan CC, et al. (2013) Intrapulmonary shear stress enhancement: A new therapeutic approach in acute myocardial ischemia. Int J Cardiol 168(4): 4199-4208. 


\section{ISSN: 2574-1241}

DOI: 10.26717/BJSTR.2020.30.004945

Sayed Nour. Biomed J Sci \& Tech Res

(c) (P) This work is licensed under Creative

Submission Link: https://biomedres.us/submit-manuscript.php

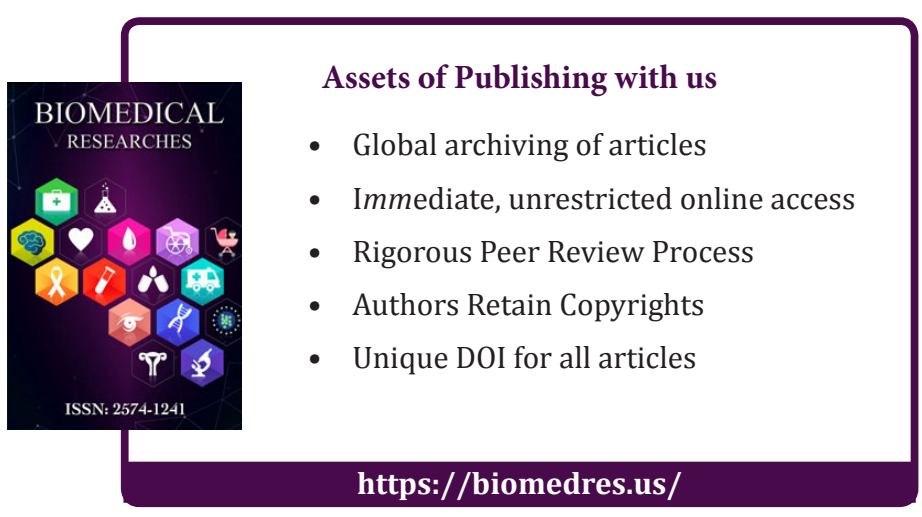

